\title{
Filamentation in Kerr media from pulsed Bessel beams
}

\author{
P. Polesana, ${ }^{1}$ M. Franco, ${ }^{2}$ A. Couairon, ${ }^{3,5, *}$ D. Faccio, ${ }^{1,5}$ and P. Di Trapani ${ }^{1,4,5}$ \\ ${ }^{1}$ CNISM and Dipartimento di Fisica, Università dell'Insubria, via Vallegio, 11, 22100 Como, Italy \\ ${ }^{2}$ Laboratoire d'Optique Appliquée, ENSTA-Ecole Polytechnique, CNRS, F-91761 Palaiseau, France \\ ${ }^{3}$ Centre de Physique Théorique, École Polytechnique, CNRS, F-91128 Palaiseau, France \\ ${ }^{4}$ Department of Quantum Electronics, Vilnius University, Sauletekio Avenue 9, LT-10222 Vilnius, Lithuania \\ ${ }^{5}$ Virtual Institute for Nonlinear Optics, Centro di Cultura Scientifica Alessandro Volta, Via Olmo Via Simone Cantoni 1, \\ 22100 Como, Italy
}

(Received 8 November 2007; revised manuscript received 21 January 2008; published 10 April 2008)

\begin{abstract}
In contrast with filamentation of ultrashort laser pulses with standard Gaussian beams in Kerr media, three different types of Bessel filaments are obtained in air or in water by focusing ultrashort laser pulses with an axicon. We thoroughly investigate the different regimes and show that the beam reshapes as a nonlinear Bessel beam which establishes a conical energy flux from the low intensity tails toward the high intensity peak. This flux efficiently sustains a high contrast long-distance propagation and easily generates a continuous plasma channel in air
\end{abstract}

\section{INTRODUCTION}

Self-focusing, self-trapping, and filamentation of intense laser beams were predicted more than 40 years ago [1]. The propagation of femtosecond laser pulses is now well known to generate intense and narrow filaments in all types of Kerr media [2,3]. Typically, in condensed media, a filament possesses a central core of a few tenths of microns and can be detected over several centimeters. In gases, the intense core from one to several hundred of microns can be detected over several hundreds of meters, by far exceeding the Rayleigh length associated with the width of the central core. Filamentation over long distances was interpreted in terms of recurrent focusing-defocusing cycles which reflect a competition between nonlinear effects [4,5]: The optical Kerr effect leads to self-focusing and overcomes diffraction as long as the peak power of the pulse exceeds the critical power $P_{\mathrm{cr}}$ $\equiv 3.77 \lambda^{2} / 8 \pi n_{0} n_{2}$ [6], where $\lambda$ is the laser wavelength, $n_{0}$ and $n_{2}$ are the linear and nonlinear index coefficients, respectively. Multiphoton absorption (MPA) sets in once the beam becomes sufficiently intense which induces three effects on the propagating pulse: First, as any high-order process, MPA limits the growth of intensity due to self-focusing [7] and induces a steplike decrease of the pulse energy which stops each refocusing cycle [8]. It was recognized that this saturation does not occur through a change of refraction index but is mediated by propagation [9]. Second, MPA can be viewed as a distributed stopper along the propagation axis which reshapes the core of the beam and tends to generate an energy flow from the outer to the central part of the beam due to the Arago-spot effect $[10,11]$. Third, MPA leads to the generation of a tenuous plasma with a density of $10^{18}-10^{19} \mathrm{~cm}^{-3}$ in condensed media [12-15], $10^{16}-10^{17} \mathrm{~cm}^{-3}$ in gases $[12,16]$. At these densities, the media remain transparent but the refraction index changes due to the plasma are sufficient to induce reshaping of the trailing part of the pulse.

\footnotetext{
*Corresponding author. couairon@cpht.polytechnique.fr
}

The concept of the energy reservoir follows from the second property and was proposed to explain the long propagation distance of filaments in air [17]. The laser energy surrounding the filament core plays the crucial role of refilling the intense central part of the filament. Multiphoton absorption occurs only in the intense and narrow core, and therefore consumes only slowly the energy of the reservoir. This promotes a recurrent nucleation of filaments and their longdistance propagation. The concept of the energy reservoir was further supported by the self-healing property of filaments [18] according to which filaments stopped by an obstacle are reconstructed after propagation of the surrounding beam over one Rayleigh length beyond the obstacle. This property was observed for filaments in water $[19,20]$ and in air $[21,22]$.

Theoretically, the refilling of the filament core by an energy flux from the periphery was explained as a spontaneous transformation of the Gaussian beam into a nonlinear conical wave $[10,20]$. A monochromatic stationary solution in the form of a nonlinear Bessel beam featured by an energy flow from the tails to the central core, called a nonlinear unbalanced Bessel beam, was then proposed as a possible attractor for the filamentation dynamics [23].

If associated with a control of the energy flux, this type of stationary solution opens up the way to several applications which require simultaneously long focal depth and high contrast such as, e.g., multiphoton microscopy, photolithography [24], micromachining [25] and channel waveguide writing with longitudinal illumination. In order to achieve the abovementioned longitudinal illumination of a bulk material with high contrast and arbitrarily long focal depth, it is of fundamental importance to understand how to promote the energy flux established during filamentation and whether it is possible to ensure an optimal energy flux. For instance, the energy in the peripheral region to a filament was shown from simulations to increase with the propagation distance indicating an energy flux opposite to the optimal flux required for applications [8]. In spite of this, preliminary results indicated that the generation of a filament from a Bessel beam would allow a controlled longitudinal illumination and deposition 
of the laser energy on the material, the energy reservoir of the Bessel beam being already that of a conical wave $[26,27]$.

In this paper, we show that powerful Bessel beams obtained by sending an ultrashort laser pulse through an axicon lead to the organization of the large Gaussian beam into a suitable energy reservoir able to sustain the required energy flux toward the core of the Bessel filament, where MPA can dissipate energy. Three types of Bessel filaments can be generated in this way, both in air and in condensed media. The first type corresponds to weakly nonlinear Bessel beams which do not undergo any losses [28]. The second type leads to a quasiperiodic deposition of energy on the material along the filament [29]. The third type is new and corresponds to a continuous longitudinal deposition of the laser energy; for instance, in air, it is materialized by a much longer plasma channel than that obtained with a standard filament formed from a Gaussian beam. We introduce suitable numerical diagnostics to monitor the energy flux established during the formation of these Bessel filaments. By means of these quantitative diagnostics, we clarify the role of the energy reservoir and determine conditions distinguishing the three regimes of Bessel filaments, in particular the third type is best suited for the above-mentioned applications, with a similar energy flow as that characterizing nonlinear unbalanced Bessel beams. We discuss the effect of plasma defocusing on the Bessel filaments which have transverse profiles similar to that of nonlinear unbalanced Bessel beams although plasma generation is not considered in these stationary solutions.

The outline of the paper is the following: Section II presents the model used for the numerical simulations of Bessel filaments and the diagnostics implemented to monitor the energy flux. Section III shows how the energy flux is shaped in standard Gaussian filaments. Section IV presents the various types of Bessel filaments obtained from simulations with various axicons and beams. Section V is finally devoted to the interpretation of the energy flux and when it is relevant, the temporal dynamics associated with the Bessel filaments.

\section{MODEL}

\section{A. Propagation of ultrashort laser pulses}

The numerical code used in this study relies on the physical model developed for the propagation of an intense pulse in noble gases or air [5,30-37], fused silica [12,13,38], and liquids [14,15,39-41].

We model the linearly polarized beam with cylindrical symmetry around the propagation axis $z$ by the envelope $\mathcal{E}$ of the electric field $\mathbf{E}$, written as $\mathbf{E}=\operatorname{Re}\left[\mathcal{E} \exp \left(i k_{0} z-i \omega_{0} t\right)\right] \mathbf{e}_{x}$, where $\mathbf{e}_{x}$ denotes the unit vector along the polarization direction, $k_{0}=k\left(\omega_{0}\right)$ and $\omega_{0}$ are the wave number and frequency of the carrier wave. The dispersion in the medium is defined by the frequency dependent wave number $k(\omega) \equiv n(\omega) \omega / c$ and the refraction index $n(\omega)$ via a Sellmeier relation. The evolution along the propagation axis $z$ of the scalar envelope $\mathcal{E}(r, t, z)$ of the electric field is described by the nonlinear envelope equation [42], expressed in the frequency domain

$$
2 i \hat{\mathcal{K}} \frac{\partial \hat{\mathcal{E}}}{\partial z}+\left[\nabla_{\perp}^{2}+\hat{\mathcal{D}}(\omega)\right] \hat{\mathcal{E}}-2 i k_{0} \mathcal{F}\{N(\mathcal{E})\}=0,
$$

where $\hat{\mathcal{E}}(r, \omega, z)=\mathcal{F}\{\mathcal{E}(r, t, z)\}, \nabla_{\perp}^{2}$ denotes the transverse Laplacian and accounts for diffraction, $\hat{\mathcal{K}}(\omega) \equiv k_{0}+\left(\omega-\omega_{0}\right) / v_{g}$ accounts for space-time focusing (see [42]), $v_{g} \equiv \partial \omega /\left.\partial k\right|_{\omega_{0}}$ denotes the group velocity, $\hat{\mathcal{D}}(\omega) \equiv k^{2}(\omega)-\hat{\mathcal{K}}^{2}(\omega)$ accounts for all dispersive terms exactly computed by means of a Sellmeier dispersion relation for the refraction index of the medium $n(\omega)$, and $\mathcal{F}\{N(\mathcal{E})\}$ denotes the time-Fourier transform of the nonlinear terms. The nonlinear effects in Eq. (1) include the optical Kerr effect with a nonlocal term corresponding to delayed Raman-Kerr response $[43,44]$, selfsteepening [through the operator $T \equiv 1+\left(i / \omega_{0}\right)(\partial / \partial t)$ in front of the Kerr term], plasma absorption, plasma defocusing, and multiphoton absorption,

$$
\begin{aligned}
N(\mathcal{E})= & i \frac{\omega_{0}}{c} n_{2} T^{2} \int_{-\infty}^{t} R(t-\tau)|\mathcal{E}(\tau)|^{2} d \tau \mathcal{E}(t)-\frac{\sigma}{2}\left(1+i \omega_{0} \tau_{c}\right) \rho \mathcal{E} \\
& -T \frac{\beta_{K}}{2}\left(1-\frac{\rho}{\rho_{\mathrm{nt}}}\right)|\mathcal{E}|^{2 K-2} \mathcal{E}
\end{aligned}
$$

The response function that accounts for both the electronic and the delayed Raman contribution to the Kerr effect may be written as [43]

$$
R(t)=\left(1-f_{R}\right) \delta(t)+f_{R} \frac{\Gamma^{2}+\Omega^{2}}{\Omega} \exp (-\Gamma t) \sin (\Omega t),
$$

where $f_{R}$ denotes the fraction of the delayed part. For plasma absorption, the cross section for inverse bremsstrahlung follows the Drude model [45] and reads as

$$
\sigma=\frac{k_{0} e^{2}}{n_{0}^{2} \omega_{0}^{2} \epsilon_{0} m} \frac{\omega_{0} \tau_{c}}{1+\omega_{0}^{2} \tau_{c}^{2}},
$$

where $\tau_{c}$ denotes the momentum transfer collision time and $m$ the electron mass for a gas or the electron-hole reduced mass for a condensed dielectric medium.

The evolution equation for the density of electrons generated by multiphoton ionization and by avalanche completes the model,

$$
\frac{\partial \rho}{\partial t}=\sigma_{K}|\mathcal{E}|^{2 K}\left(\rho_{\mathrm{nt}}-\rho\right)+\frac{\sigma}{U_{i}} \rho|\mathcal{E}|^{2},
$$

where $\rho_{\mathrm{nt}}, U_{i}, K \equiv\left\langle\frac{U_{i}}{\hbar \omega_{0}}+1\right\rangle, \sigma_{K}$, and $\beta_{K} \equiv \sigma_{K} K \hbar \omega_{0} \rho_{\mathrm{nt}}$ denote the density of neutral atoms or molecules, the ionization potential, the number of photons involved in the process, the cross sections for multiphoton ionization and multiphoton absorption, respectively.

Finally the input fields are pulsed Gaussians beams with a spatial phase modeling their passage through the axicon,

$$
\mathcal{E}(r, t, 0)=\mathcal{E}_{0} \exp \left(-\frac{r^{2}}{w_{0}^{2}}-\frac{t^{2}}{t_{p}^{2}}-i k_{0} r \sin \theta\right),
$$

which generates a Bessel beam $\mathcal{E}\left(r, t, z_{\mathrm{ax}}\right) \propto J_{0}\left(k_{0} r \sin \theta\right)$ after linear propagation over a distance $z_{\mathrm{ax}}=w_{0} / 2 \tan \theta$, where the Bessel angle $\theta \simeq \gamma\left(n_{\mathrm{ax}}-1\right)$ is obtained from the Snell law $\sin (\gamma+\theta)=n_{\mathrm{ax}} \sin (\gamma)$ in the limit of small angles, and $\gamma$ and $n_{\mathrm{ax}}$ denote the base angle and the refraction index of the axicon (see Fig. 1). Notably, the input field (5) is similar to that generated by a hologram and the pulse moves at velocity $v_{g} \cos \theta$ [46]. We investigated the formation of Bessel filaments in air and in water. In the case of water contained in a 


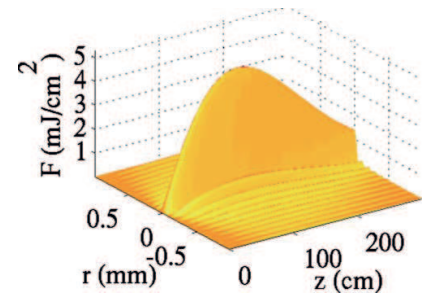

FIG. 1. (Color online) Fluence distribution obtained by propagation of a low energy, $50 \mathrm{fs}, w_{0}=1 \mathrm{~cm}$ pulse through an axicon of base angle $0.5^{\circ}$.

glass cell, the refraction at the transition air-glass-water is accounted for by using the Bessel angle $\theta \simeq \gamma\left(n_{\mathrm{ax}}-1\right) / n_{\text {water }}$. The temporal half-width $t_{p}$ of the pulse is linked to the temporal full width at half-maximum (FWHM) duration by $\tau_{\mathrm{FWHM}}=t_{p} \sqrt{2 \ln 2}$. The input power is computed from the energy $E_{\text {in }}$ and pulse duration $P_{\text {in }}=E_{\text {in }} / t_{p} \sqrt{\pi / 2}$. The input intensity is computed from the input power and the transverse waist $w_{0}$ as $\mathcal{E}_{0}^{2}=2 P_{\text {in }} / \pi w_{0}^{2}$.

Simulation results will be presented for Bessel filaments in water and in air with the parameters indicated in Table I modeling experimental situations with either a Nd:glass laser for water $\left(\lambda_{0}=527 \mathrm{~nm}\right)$ or a Ti:S laser for air $\left(\lambda_{0}\right.$ $=800 \mathrm{~nm}$ ). Some coefficients are directly introduced in the model. Others such as $P_{\text {cr }}$ or $k_{0}^{\prime \prime}=\left.\frac{\partial^{2} k}{\partial \omega^{2}}\right|_{\omega_{0}}$ do not directly enter in the model but are given to facilitate the estimation of the relevance of corresponding physical effects.

\section{B. Diagnostics for the energy exchanges}

In the present paper we will introduce relevant numerical diagnostics which give an insight in the physics of the energy

TABLE I. Parameters used in the simulations and in the analysis of results. The coefficients for water were determined from the procedure of Ref. [14].

\begin{tabular}{lcc}
\hline \hline & Air, $800 \mathrm{~nm}$ & Water, $527 \mathrm{~nm}$ \\
\hline$\tau_{\mathrm{FWHM}}(\mathrm{fs})$ & 50 & 200 \\
$n_{2}\left(\mathrm{~cm}^{2} / \mathrm{W}\right)$ & $3.2 \times 10^{-19}$ & $2.7 \times 10^{-16}$ \\
$P_{\mathrm{cr}}$ & $3 \mathrm{GW}$ & $1.2 \mathrm{MW}$ \\
$f_{R}$ & 0.5 & 0 \\
$\Gamma^{-1}(\mathrm{fs})$ & 70 & \\
$\Omega(\mathrm{THz})$ & 16 & \\
$k_{0}^{\prime \prime}\left(\mathrm{fs}^{2} / \mathrm{cm}\right)$ & 0.2 & 560 \\
$U_{i}(\mathrm{eV})$ & 12.1 & 7.1 \\
$K$ & 8 & 4 \\
$\sigma_{K}\left(\mathrm{~s}^{-1} \mathrm{~cm}^{2 K} \mathrm{~W}^{-K}\right)$ & $3.7 \times 10^{-96}$ & $2.4 \times 10^{-42}$ \\
$\beta_{K}\left(\mathrm{~cm}^{2 K-3} \mathrm{~W}^{1-K}\right)$ & $1.8 \times 10^{-94}$ & $2.4 \times 10^{-37}$ \\
$\tau_{c}(\mathrm{fs})$ & 350 & 3 \\
$m$ & $m_{e}$ & $0.5 m_{e}$ \\
$\sigma\left(\mathrm{cm}^{2}\right)$ & $5 \times 10^{-20}$ & $4 \times 10^{-18}$ \\
$\rho_{\mathrm{nt}}\left(\mathrm{cm}^{-3}\right)$ & $5 \times 10^{18}$ & $7 \times 10^{22}$ \\
$\gamma$ & $5^{\circ}$ & $2^{\circ} 50^{\prime}$ \\
$n_{\mathrm{ax}}$ & 1.51 & 1.52 \\
$\theta\left(\mathrm{mrad}^{\circ}\right.$ & 45 & 19 \\
\hline \hline
\end{tabular}

reservoir. To this aim, we will monitor the power, the current, energy, losses, and flux, associated with a volume partially or fully containing the filament. We follow the evolution of these quantities along the propagation distance and we shall evidence the advantage of generating filaments with Bessel beams (over Gaussian beams) for the above-mentioned applications.

Most simulation results presented in this work do not critically depend on high-order dispersive terms and can be retrieved by using a small $\omega-\omega_{0}$ expansion of $\hat{\mathcal{D}}(\omega)$ $\simeq k_{0}^{\prime \prime} k_{0}\left(\omega-\omega_{0}\right)^{2}$ in Eq. (1). Although this approximation was not performed in the simulations, we will use it to simplify the presentation of the numerical diagnostics for the energy flux. Thus Eq. (1) can be written in the time domain by using the retarded time $t \equiv t_{\mathrm{lab}}-z / v_{g}$,

$$
\frac{\partial \mathcal{E}}{\partial z}=\frac{i}{2 k_{0}} \nabla_{\perp}^{2} \mathcal{E}-i \frac{k_{0}^{\prime \prime}}{2} \frac{\partial^{2} \mathcal{E}}{\partial t^{2}}+N(\mathcal{E}),
$$

where we also introduced $\hat{K} \simeq k_{0}$ and $T \simeq 1$ (space-time focusing and self-steepening neglected).

In order to monitor the energy flow along the propagation axis, we systematically performed diagnostics on different quantities defined as follows:

(i) The partial power contained in a cylinder of radius $R$,

$$
P_{R}(z, R, t)=\int_{0}^{R}|\mathcal{E}(z, r, t)|^{2} 2 \pi r d r .
$$

(ii) The transverse field current which is negative when the flux is inward to the cylindrical surface of radius $r$,

$$
j_{\perp}(z, r, t)=\frac{1}{2 i}\left(\mathcal{E}^{*}(z, r, t) \frac{\partial \mathcal{E}}{\partial r}-\mathcal{E}(z, r, t) \frac{\partial \mathcal{E}^{*}}{\partial r}\right) .
$$

As an example, the transverse current for a Gaussian pulse focused by a lens,

$$
\mathcal{E}(r)=\exp \left(-\frac{r^{2}}{w_{0}^{2}}-i \frac{k r^{2}}{2 f}\right),
$$

where $f$ denotes the curvature of the wave front, reads as

$$
j_{\perp}(r)=-2|\mathcal{E}(r)|^{2} \frac{k r}{f} .
$$

The transverse field current is inward (negative), for a focusing beam $(f>0)$, and outward for a defocusing beam. This result can be extended in the case of the Kerr effect: When the power exceeds $P_{\mathrm{cr}}$ the phase curvature becomes positive upon propagation and the transverse current becomes negative.

(iii) The energy density for the nonlinear losses due to multiphoton absorption and plasma absorption,

$$
\begin{gathered}
q_{\mathrm{MPA}}(z, r, t)=\beta_{K}|\mathcal{E}(z, r, t)|^{2 K}\left[1-\rho(z, r, t) / \rho_{\mathrm{nt}}\right], \\
q_{\mathrm{PA}}(z, r, t)=\sigma \rho(z, r, t)|\mathcal{E}(z, r, t)|^{2} .
\end{gathered}
$$

The evolution equation for the partial power is obtained by integration of Eq. (6), 


$$
\begin{aligned}
\frac{\partial P_{R}}{\partial z}= & -\frac{2 \pi}{k_{0}} R j_{\perp}(z, R, t)-k_{0}^{\prime \prime} \int_{0}^{R} \operatorname{Im}\left(\mathcal{E} \frac{\partial^{2} \mathcal{E}^{*}}{\partial t^{2}}\right) 2 \pi r d r \\
& -\int_{0}^{R} q_{\mathrm{MPA}}(z, r, t) 2 \pi r d r-\int_{0}^{R} q_{\mathrm{PA}}(z, r, t) 2 \pi r d r .
\end{aligned}
$$

Similar diagnostics related to the partial energy, contained in a cylinder of radius $R$ and defined as

$$
E_{R}(z, R)=\int_{-\infty}^{+\infty} \int_{0}^{R}|\mathcal{E}(z, r, t)|^{2} 2 \pi r d r d t
$$

are obtained by integration of the previously defined quantities over time.

(iv) The time-integrated flux of the transverse field current is defined as

$$
J_{\perp}(z, R)=2 \pi R \int_{-\infty}^{+\infty} j_{\perp}(z, R, t) d t .
$$

(v) The density of energy absorbed by multiphoton processes reads as

$$
Q_{\mathrm{MPA}}(z, R)=\int_{-\infty}^{+\infty} \int_{0}^{R} q_{\mathrm{MPA}}(z, r, t) 2 \pi r d r d t,
$$

and a plasma absorption leads to a similar expression for $Q_{\mathrm{PA}}$.

The evolution equation for the partial energy then reads as

$$
\frac{\partial E_{R}}{\partial z}=-\frac{J_{\perp}(z, R)}{k_{0}}-Q_{\mathrm{MPA}}-Q_{\mathrm{PA}} .
$$

Multiphoton absorption usually prevails over plasma absorption when the beams are not too tightly focused, which is the case of our calculations in this paper. The above equation thus shows that multiphoton absorption can be compensated by an inward (negative) energy current.

\section{FILAMENTS WITH ULTRASHORT PULSES AND GAUSSIAN BEAMS}

\section{A. Pulse splitting dynamics in filamentation of Gaussian beams}

Standard filamentation features may be recalled by considering filamentation in water, which was simulated for a $527 \mathrm{~nm}$ input pulse with duration $\tau_{\mathrm{FWHM}}=200$ fs, energy $E_{\text {in }}=3 \mu \mathrm{J}$ and width of the Gaussian beam $w_{0}=100 \mu \mathrm{m}$. Figure 2 shows the evolution along the propagation axis of the beam radius measured as the half-width at half-maximum of the fluence distribution $F(r, z)=\int_{-\infty}^{+\infty}|\mathcal{E}(z, r, t)|^{2} d t$. Although the nearly constant narrow diameter seems to indicate a stationary propagation within the filament, this diagnostic masks complex dynamics in space and time, which involve recurrent pulse-splitting events induced by successive Kerr self-focusing stages stopped by strong multiphoton absorption of the intense central part of the pulse. Figure 2(b) displays the temporal dynamics resulting from these phenomena. Each splitting event leads to a forklike pattern, where
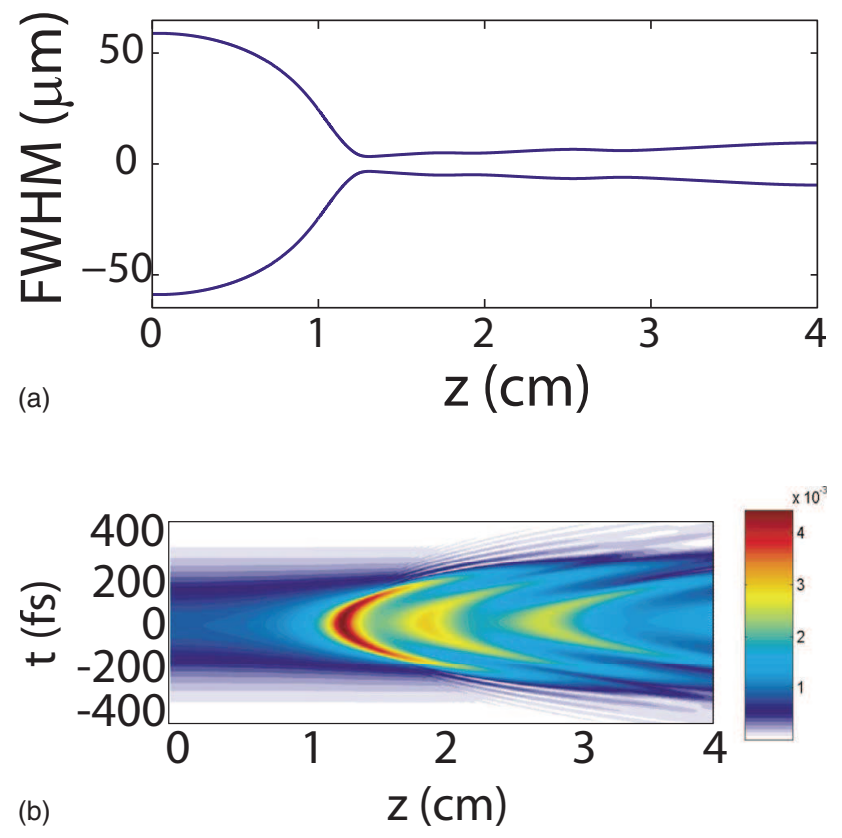

FIG. 2. (Color online) (a) Full width at half-maximum of the fluence distribution for a $527 \mathrm{~nm}, 200 \mathrm{fs}, 3 \mu \mathrm{J}$ laser pulse undergoing filamentation in water. (b) Evolution of the on-axis intensity profiles vs propagation distance showing recurrent splitting dynamics.

the two arms of a fork correspond to the leading (negative times) and trailing (positive times) split pulses.

\section{B. Energy flux in filaments}

Several papers have shown that these splitting events are associated with the generation of conical waves which are $\mathrm{X}$-shaped in space and time $(r, t)$ with one or two intense and narrow peaks surrounded by weaker but weakly localized tails [22,39-41,47-50]. One of the most convincing evidence supporting the generation of an $X$ wave consists in the proposition that the extended tails surrounding the filament naturally explain the physics of the energy reservoir which refills the central peak. Experiments showed that a filament reconstructs its central peak when it is absorbed by a stopper $[19,20]$ : This phenomena can be explained only by the regenerative property of the conical waves. This evidence can be detailed with a numerical analysis of the energy redistribution during propagation.

Simulations allowed us to easily analyze this property by monitoring the field current established during filamentation. For the same filamentation numerical experiment as that reported in Fig. 2, we selected the near field at the propagation distances $z=1.4,1.5,1.6$, and $1.7 \mathrm{~cm}$ and computed the transverse field current. The results are reported in Fig. 3: At the nonlinear focus [Fig. 3(a)] the field current is inward due to the Kerr self-focusing effect. When pulse splitting occurs, the highest field currents remain centered on the split pulses and the spatiotemporal distribution of the current is $\mathrm{X}$ shaped as visible in Figs. 3(b)-3(d). In order to describe the refilling mechanism, the current dynamics was linked to the nonlinear 


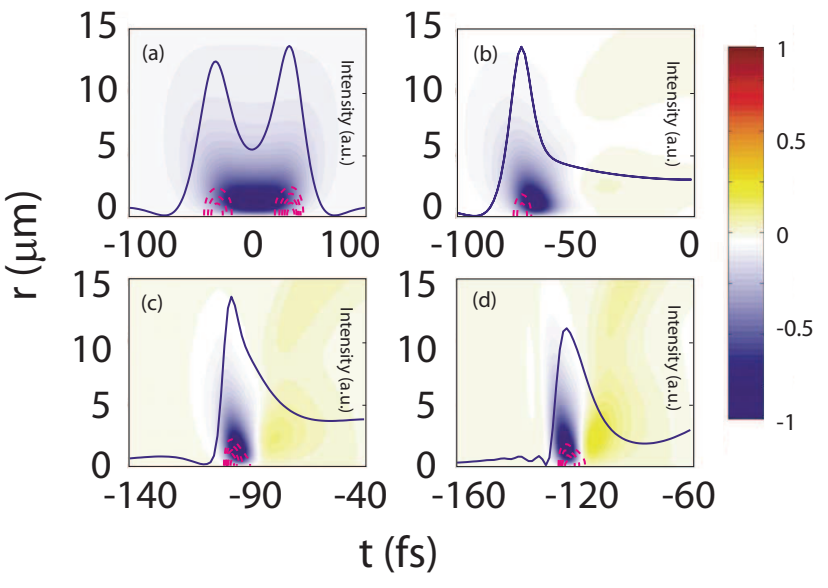

FIG. 3. (Color online) Map of the field current (normalized) as a function of time (horizontal axis) and radius (vertical axis) of the filament at different propagation distances. On each map is superimposed the map in radial and temporal coordinates of the nonlinear losses in dashed isointensities and the intensity profile at $r=0$ as a function of time. Propagation distances are from (a) to (d) $z=1.4$, $1.5,1.6,1.7 \mathrm{~cm}$.

losses [i.e., mainly multiphoton absorption $\left.q_{\mathrm{MPA}}(z, r, t)\right]$ which tend to dissipate the peak of the filament. Figure 3 displays the map of the losses as contour lines (dashed curves) superimposed on the field flux map and on the plot of the axial pulse intensity (solid curves). Figures 3(b)-3(d) show a time-window centered on the leading part of the pulse; the (not shown) trailing pulse exhibits qualitatively symmetric features. Of particular interest, the leading peak of the pulse carries always an inward current, while after the trailing edge the current is outward. Moreover, losses are always concentrated in the peak of the $X$ wave where the sign of the current is negative: In this way the $X$ wave ensures a continuous refilling of the losses, thus establishing a regime clearly robust to dissipations.

\section{DIFFERENT REGIMES OF BESSEL FILAMENTATION}

Three different regimes of Bessel filamentation can be distinguished from our numerical simulations and previous literature

(i) The weakly nonlinear Bessel filamentation regime: It is obtained by slightly increasing the power of the input Gaussian beam from the linear propagation regime until the power contained in the central peak of the Bessel beam is sufficient to induce self-focusing but insufficient to induce collapse or significant MPA and electron density [28]. The peak intensity of the resulting self-guided beam exhibits oscillations simply characterizing a recurrent Kerr compression of the transverse rings of the Bessel beam formed beyond the axicon. The reader is referred to Ref. [28] for a detailed analysis of this regime.

(ii) Starting from already formed Bessel beams, Gaižauskas et al. [29] have shown the formation of very regular discrete damage traces in the bulk of a nonlinear sample. The main difference with regime (i) is the power content of the
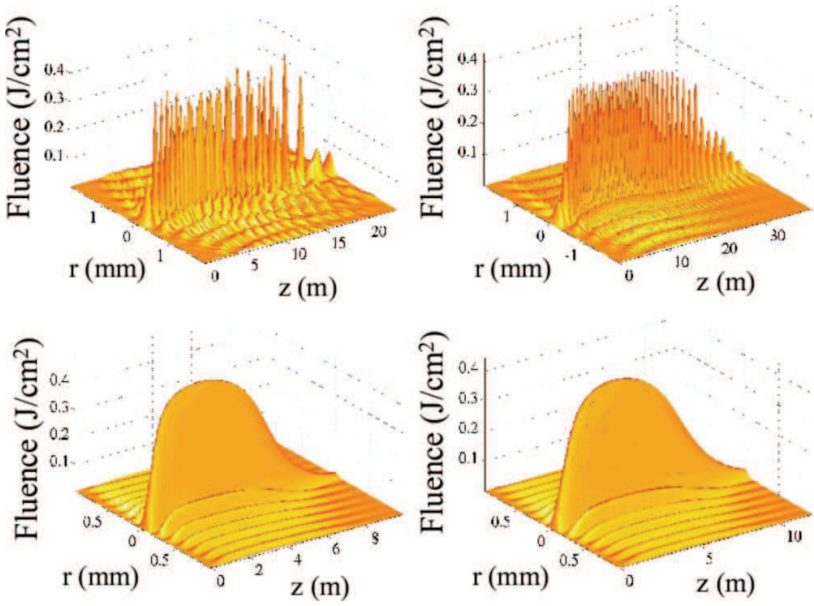

FIG. 4. (Color online) Fluence distribution obtained by sending a Gaussian beam of power $P_{\text {in }}=100 P_{\text {cr }}$ and waist $w_{0}$ through an axicon of base angle $\gamma$. First column, $w_{0}=15 \mathrm{~mm}$; second column, $w_{0}=20 \mathrm{~mm}$; first line, $\gamma=0.1^{\circ}$; second line, $\gamma=0.3^{\circ}$.

central peak of the Bessel beam, which is sufficient to induce collapse, and hence, MPA and damage. Hereafter, we will refer to this regime as that of unsteady Bessel filamentation.

(iii) Polesana et al. [51] have shown that the input conditions in the nonlinear sample can either promote or prevent the temporal and spectral dynamics of the pulse [51]: If the Gaussian beam propagates through the axicon and reshapes into a Bessel beam before entering the nonlinear sample, strong self-focusing of the central peak of the Bessel beam occurs which in turn triggers MPA, induces a fast reduction of the peak intensity and the process can occur again while Kerr self-focusing of the external ring periodically brings energy to the core. In contrast, if the nonlinear sample is in contact with the axicon, the Gaussian beam smoothly reshapes into a nonlinear unbalanced Bessel beam [23,51]. Below, the latter case will be referred to as the steady Bessel filamentation regime and will be thoroughly investigated by simulations in air.

Figure 4 shows a comparison between the fluence distributions obtained for Bessel filaments generated in air with different beams and axicons. It is clear from this panel that a transition between the unsteady (shown in the first line) and the steady (shown in the second line) Bessel filamentation regimes exists when the base angle of the axicon is increased. In the unsteady Bessel filamentation regime, the quasiperiodic intense spots along the propagation axis cover a distance about 5 times larger than that obtained for the continuous light channel generated in the steady Bessel filamentation regime with the same beam power and waist. The regularity of the quasiperiodic light channel is improved for the larger input beam waist. These regimes are associated with a plasma channel of density of a few $10^{16} \mathrm{~cm}^{-3}$ due to ionization of oxygen molecules (data not shown). The simultaneous excitation of nitrogen molecules should gives rise to a fluorescence track. The continuity of both the plasma channel and the fluorescence track follows that of the intense light channel.

Figure 5 shows three examples of fluence distribution and associated long plasma channels shaped as very flat plateaus 

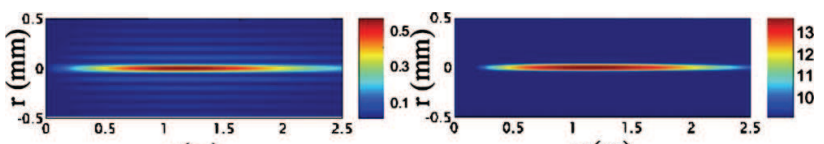

$\mathrm{z}(\mathrm{m})$

(m)
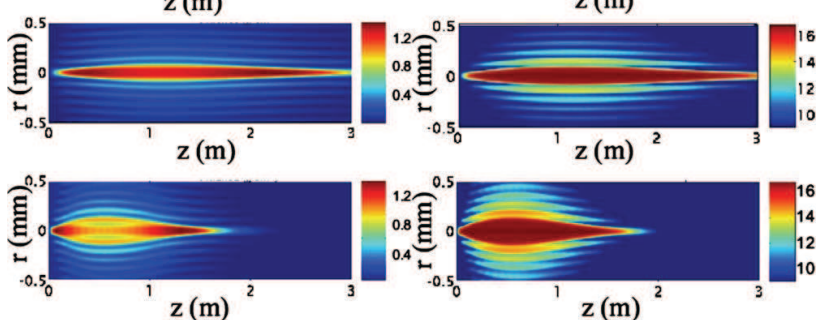

FIG. 5. (Color online) Fluence distribution (first column) and plasma channels (second column) generated by Bessel filamentation. The color bar units are in $\mathrm{J} / \mathrm{cm}^{2}$ for the fluence (left-hand column) and in decades $\left(16 \equiv 10^{16} \mathrm{~cm}^{-3}\right)$ for the electron densities (right-hand column). The parameters of the input pulses sent through the axicon of base angle $\gamma=0.5^{\circ}$ are $\tau_{\mathrm{FWHM}}=50$ fs. First line, $E_{\text {in }}=1.44 \mathrm{~mJ}, w_{0}=10 \mathrm{~mm}$; second line, $E_{\text {in }}=14.4 \mathrm{~mJ}, w_{0}$ $=10 \mathrm{~mm}$; third line, $E_{\text {in }}=14.4 \mathrm{~mJ}, w_{0}=5 \mathrm{~mm}$.

of constant density. Naturally, Fig. 5 should not be interpreted as representing the light and plasma channel at a given instant but as the traces left by the propagating pulses on the medium. For instance, the electron densities shown in Fig. 5 represent the largest plasma densities reached for each propagation distance. This steady Bessel filamentation is obtained for a wide range of input parameters and is robust with respect to an increase of the input pulse energy. It is interesting to note that the fluence distributions as well as the plasma channels obtained in Bessel filaments can exhibit a Bessel-like profile which reflect the action of nonlinearities on the profile of the axicon-focused input beam (radial compression and loss of contrast of the Bessel oscillations [27]). However, the detailed intensity distribution in space and time exhibits other remarkable features not simply describable by referring to the formation of nonlinear Bessel profiles.

For instance, Fig. 6 shows the evolution of the temporal profiles of the on-axis intensity distribution. Limited temporal dynamics are observable from these profiles: The on-axis intensity remains bell shaped and inspection of the associated spectra (not shown) indicates also a very limited con-

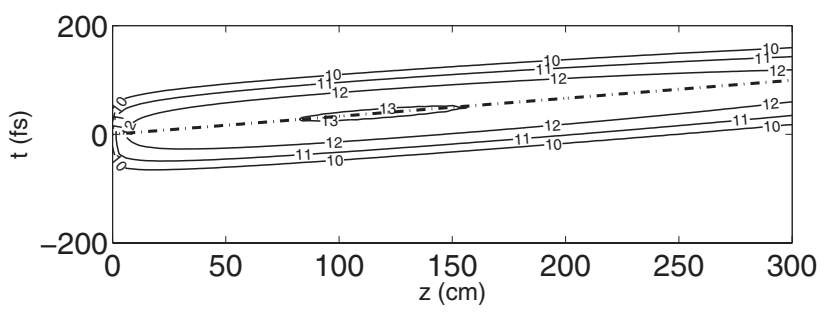

FIG. 6. Evolution of the temporal profiles of the on-axis intensity distribution along the propagation distance for the $E_{\text {in }}$ $=1.44 \mathrm{~mJ}, w_{0}=10 \mathrm{~mm}, \tau_{\mathrm{FWHM}}=50 \mathrm{fs}$ input pulse focused with the $\gamma=0.5^{\circ}$ axicon. The numbers label the intensity levels (13 $\equiv 10^{13} \mathrm{~W} / \mathrm{cm}^{2}$ ) and the dashed line marks the predicted velocity of the Bessel pulse.
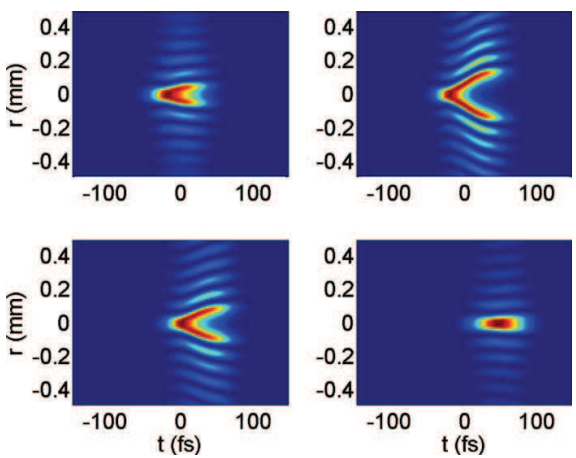

FIG. 7. (Color online) Evolution of the intensity distribution in space and time during the process of Bessel filamentation. Input pulse: $E_{\text {in }}=14.4 \mathrm{~mJ}, w_{0}=5 \mathrm{~mm}, \tau_{\mathrm{FWHM}}=50 \mathrm{fs}, \gamma_{\mathrm{ax}}=0.5^{\circ}$. Propagation distances: $z=10 \mathrm{~cm}$ (top left), $z=50 \mathrm{~cm}$ (top right), $z$ $=100 \mathrm{~cm}$ (bottom left), $z=150 \mathrm{~cm}$ (bottom right).

tinuum generation. The peak intensity propagates at a group velocity which is given by $\left.v_{g}\right|_{\omega_{0}} \cos \theta$, where $\theta$ is the Bessel angle. This diagnostic, however, masks a slightly more complex reshaping in space and time.

Figure 7 presents in details the evolution of the intensity distribution in the Bessel filament. An important reshaping occurs due to the effect of plasma defocusing. While the transverse profiles exhibit the ringlike features of Bessel beams in the leading part of the pulse, the trailing part is clearly shaped as a cone in the space-time domain.

The steady Bessel filamentation regime where the interaction with the medium and the generation of a plasma is achieved continuously over a long distance is the most interesting for applications. For instance, a steady Bessel filament in air could lead to an enhanced $\mathrm{THz}$ generation in the forward direction [52-55]. Amplification of a Bessel filament in a suitable medium should be easier to perform than that of a standard filament [56]. Since there might be some uncertainty in the parameters used in the simulations, notably in the determination of the multiphoton absorption coefficients, we varied the multipoton absorption cross section $\sigma_{K}$ while other parameters were kept constant and we found a transition from the steady Bessel filamentation regime to the weakly nonlinear filamentation regime when $\sigma_{K}$ was decreased below a certain threshold $\left(\sigma_{8}=3.7\right.$ $\times 10^{-98} \mathrm{~s}^{-1} \mathrm{~cm}^{16} \mathrm{~W}^{-8}$ for $K=7$ photons or below $\sigma_{7}=2.3$ $\times 10^{-83} \mathrm{~s}^{-1} \mathrm{~cm}^{14} \mathrm{~W}^{-7}$ for $K=7$ photons). We have shown numerically that a transition from the steady to the unsteady Bessel filamentation regimes occurs when the base angle of the axicon is decreased which will be discussed in the next section.

\section{UNSTEADY BESSEL FILAMENTATION AND TRANSITION TO THE STEADY Bessel FILAMENTATION REGIME}

\section{A. Space-time dynamics of the unsteady Bessel filaments}

A transition between the steady to the unsteady Bessel filamentation regimes was also observed in simulations of Bessel filamentation in a water cell with ultrashort laser 


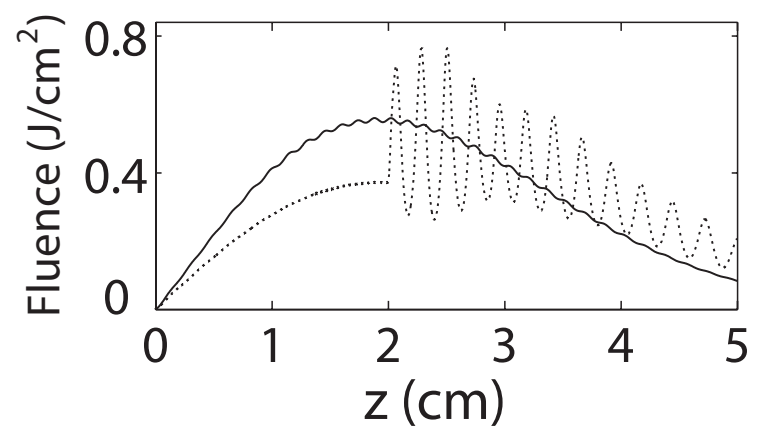

FIG. 8. Peak fluence versus propagation distance resulting from simulation. Dashed curve: Unsteady Bessel filament [regime (ii)] obtained with an abrupt linear-nonlinear transition at $z_{\mathrm{ax}}=20 \mathrm{~mm}$. Solid curve: Steady Bessel filament [regime (iii)] obtained with a soft transition to the nonlinear regime. Input pulse: $527 \mathrm{~nm}, E_{\text {in }}$ $=10 \mu \mathrm{J} \tau_{\mathrm{FWHM}}=200 \mathrm{fs}, w_{0}=0.8 \mathrm{~mm}, \gamma=2.83^{\circ}$.

pulses sent through an axicon when the position of the entrance face of the cell was shifted from the tip of the axicon [51]. The peak power of the input Gaussian beam was above the self-focusing threshold for water but below that for air. For a 5-cm-long water cell in contact with the axicon, the axicon focuses the Gaussian beam into a Bessel filament within the cell. The steady Bessel filament then forms smoothly (solid curve in Fig. 8) and was shown to undergo reshaping into structures identified as nonlinear unbalanced Bessel beams [23,51]. In contrast, for a 3-cm-long water cell placed $2 \mathrm{~cm}$ away from the tip of the axicon, a Bessel beam forms in air and then enters the water cell abruptly, i.e., with peak intensity and power sufficient to immediately trigger nonlinear effects. This results in the unsteady Bessel filamentation regime (dashed curve in Fig. 8) with strong oscillations of the beam features around those of the potential nonlinear unbalanced Bessel beams attractors in the form of nonlinear unbalanced Bessel beams, and an associated temporal and spectral dynamics detailed below.

Figure 9(a) shows the intensity map for the near-field of the unsteady Bessel filament at the propagation distance of $1.4 \mathrm{~cm}$ in the nonlinear medium $(3.4 \mathrm{~cm}$ on Fig. 8). The simulation shows that recurrent pulse splitting events are transmitted from the central peak of the Bessel-like solution to the external rings. Figure 9(a) shows a central peak just before splitting and a couple of pre- and post-subpulses, which actually come from the occurrence of a previous pulse splitting event. The first ring is also populated by two couples of subpulses. The near-field dynamics which reshapes the central peak and each Bessel ring can be summarized in three steps which occur cyclically: (i) Pulse splitting in time of the central peak; (ii) separation of the split pulses; (iii) reconstruction of the central peak. These dynamics reshape each Bessel ring with some delay with respect to the center owing to the energy flux from the ring toward the center established when the central peak is reconstructed.

The angularly resolved spectra associated to this cyclical dynamics also present some remarkable features which may be used as signatures for an experimental detection of the number of pulse splitting events [51]. Figure 9(b) displays
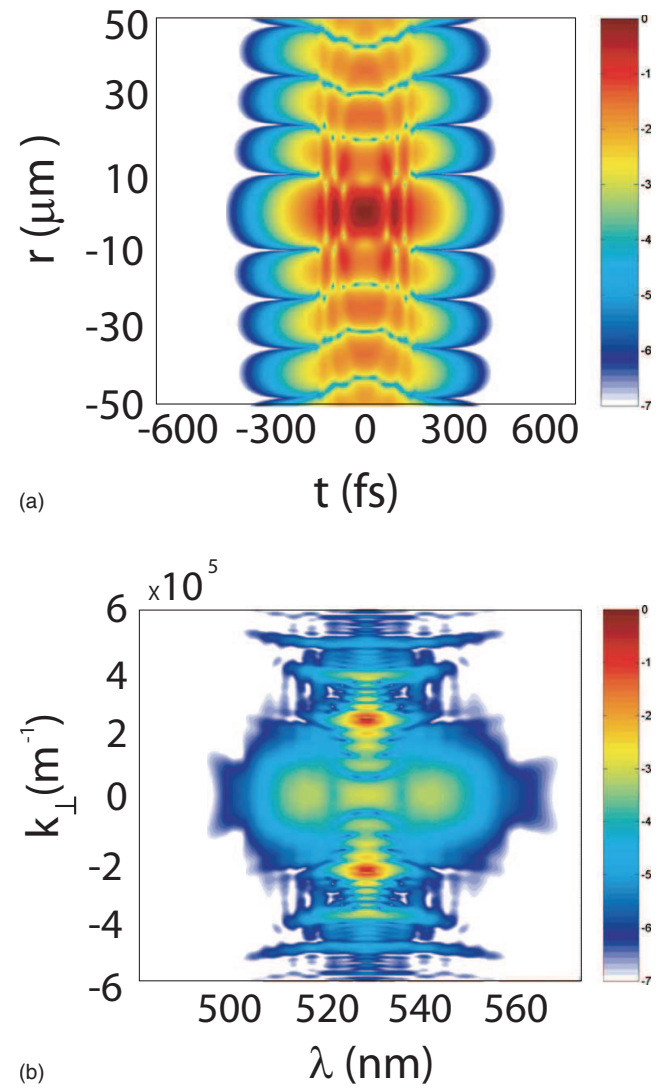

FIG. 9. (Color online) (a) Detailed near-field $I(r, t)$ for the unsteady Bessel filament in water at propagation distance $z=1.4 \mathrm{~cm}$. The temporal substructures result from cyclical splitting events. (b) Angularly resolved spectra obtained from simulation of unsteady Bessel filaments at the same propagation distance.

the numerically computed angularly resolved spectrum of the field at the same propagation distance as in Fig. 9(a). The relevant features are the axial continuum, the secondary ring $\left(k_{\perp}=3.8 \times 10^{5} \mathrm{~m}^{-1}\right)$, and the paired horizontal bands $\left(k_{\perp}\right.$ $\left.= \pm 2 \times 10^{5} \mathrm{~m}^{-1}, \pm 1.5 \times 10^{5} \mathrm{~m}^{-1}, \pm 0.5 \times 10^{5} \mathrm{~m}^{-1}\right)$. The axial emission consists in three parts resulting from a splitting of the broad continuum. The arms of the cross-shaped central part were formed by a splitting in time of the pulse and give rise to the generation of an additional paired band. Each pulse splitting in time is associated with a stretching of the central part of the continuum which is finally split into three parts and generates an additional paired band. The number of paired bands thus corresponds to the number of splitting events.

\section{B. Interpretation of the periodicity of unsteady Bessel filaments}

The complex splitting dynamics occurring in the unsteady Bessel filaments can be interpreted as the destructive interference between the conical wave and a tightly localized pulse superimposing with the Bessel central peak and propagating longitudinally. Nonlinear effects play a role mainly in the intense central peak of the Bessel beam, where pulse 
splitting results from the interplay between Kerr selfcompression and multiphoton absorption. Group velocity dispersion of course plays also a role in preventing or smoothing the development of shocks on the intense split pulses. At the moment of pulse splitting, there is a phase mismatch of $\pi$ between the Bessel beam with cone angle $\theta$ and the localized pulse. During propagation over a distance $z$, a longitudinal phase mismatch $\Delta \phi=k_{0}(1-\cos \theta) z$ accumulates between the two components, due to the different longitudinal components of the wave vector in the Bessel beam and in the axial part of the beam. This means that at a distance $z=\pi / k(1-\cos \theta)$, the phase mismatch reaches $2 \pi$ leading to a constructive interference and to the reconstruction of the central peak. Pulse splitting then occurs with a periodicity

$$
L=2 \pi / k(1-\cos \theta) .
$$

For the Bessel angle $\theta=19.3 \mathrm{mrad}$ used in our simulations, the predicted periodicity is $L=2.1 \mathrm{~mm}$, in agreement with that obtained in the simulation shown in Fig. 8.

The reconstruction of the central peak is a well-known feature of the Bessel beam which found interesting applications [57]. If the central peak of a Bessel beam is absorbed by a stopper, it is indeed reconstructed due to the continuous conical flux of energy ensured by the tails. Due to the conical geometry of the wave, a depletion of the rings also takes place, i.e., the energy flux depletes the first ring to refill the central peak and this depletion process is then transferred to the external neighboring rings. The same phenomena holds also for pulsed Bessel beams as in the case of our simulations of Bessel filaments: Multiphoton absorption depletes the central peak and leads to its temporal splitting; the conical tails reconstruct a new central peak, which seemingly transfers the pulse splitting to the first ring. In fact, the first ring seems to be split in time because the energy flux reconstructing the central peak is localized in the central time slices of the first ring. A new pulse splitting event then takes place in the center. The regular occurrence of pulse splitting events accompanied by energy transfer to the axial part of the beam, progressively modifies also the radial profile. These changes are at the origin of the generation of couples of paired bands in the far field, as reported in Fig. 9(b). It follows from this observation that the number of splitting events that occurred in the central peak of the Bessel filament can be determined by a simple inspection of the number of paired bands in the far field.

\section{Nonlinear losses and energy flux in unsteady Bessel filaments}

We analyzed quantitatively the energy flux in the case of unsteady Bessel filaments, i.e., for simulations of the abrupt input of the Bessel beam in the water cell. The arrest of self-focusing of the central peak of the Bessel beam by MPA features recursive pulse splitting events very similar to the case of the filament generated with a Gaussian beam. We have analyzed the evolution of the field current along the propagation distance.

The calculated transverse field current is presented in Fig. 10 at four distances highlighting a pulse splitting event. The
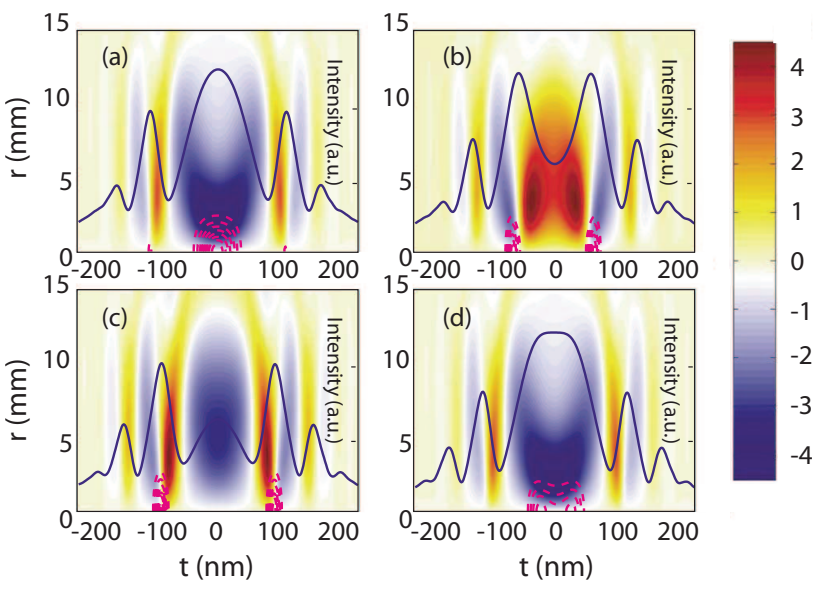

FIG. 10. (Color online) Maps of the field current as a function of time (horizontal axis) and radius (vertical axis) of the filament generated by a $200 \mathrm{fs}$ Bessel pulse in abrupt input conditions. On each map is superimposed the map of MPA in dashed isointensities and the intensity profile at $r=0$ as a function of time. Each subfigure corresponds to different propagation distances: From (a) to (d) $z$ $=1.38,1.47,1.56,1.65 \mathrm{~cm}(+2 \mathrm{~cm}$ in air before the abrupt injection).

maps of the nonlinear losses [the contours of $\left.q_{\mathrm{MPA}}(r, t)\right]$ are shown with dashed curves and the axial temporal profiles with the solid curves. At $z=3.4 \mathrm{~cm}$ [Fig. 10(a)], a new cycle is about to start with a pulse splitting: A clear inward field current is established around $t=0$, where MPA is important; besides the central peak, other peaks formed by previous splitting events are present. In Figs. 10(b) and 10(c) the central peak has split and two new subpulses separate from each other and propagate away from the central time slice $t=0$. An inward field current points toward each split peak where nonlinear losses consumes energy. In Fig. 10(d), the split pulses have traveled farther from the center $t=0$ while the central peak was reconstructed by the dynamics detailed in Fig. 9. The transverse field current, i.e., the energy flux, becomes again similar to that in Fig. 10(a). This analysis thus shows that the unsteady Bessel filaments are sustained by an energy flux toward the center which refills the sinks where MPA occurs. The process is somewhat similar to that occurring in filaments formed with Gaussian beams except that the energy flux in Bessel filaments features a conical wave different from an $X$ wave.

\section{Nonlinear losses and energy flux in steady Bessel filaments}

For steady Bessel filaments obtained when the beam enters the nonlinear Kerr medium just after the axicon, the temporal dynamics are much less relevant than for unsteady Bessel filaments. The energy density current, i.e., the timeintegrated transverse field current through a cylinder of radius $R, J_{\perp}(z, R)$ [see Eq. (15)], is then considered to eliminate the redundant dependence of the energy flux in that coordinate. Evidently an inward energy current gives rise to a negative flux. The energy density flux calculated in the case of the steady Bessel filament is shown in Fig. 11. Figure 

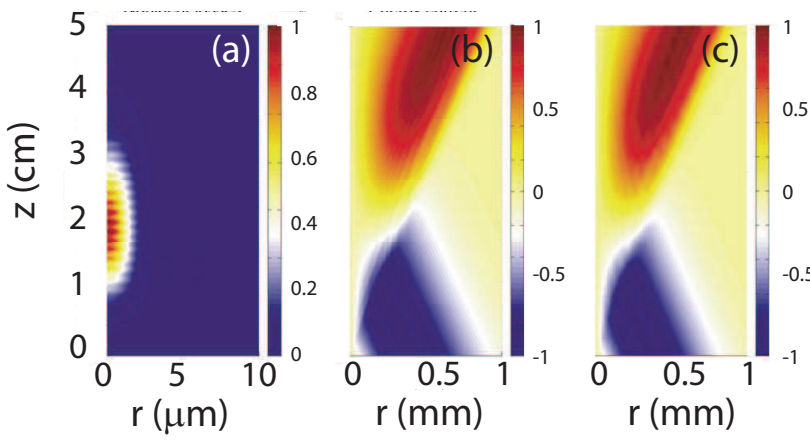

FIG. 11. (Color online) (a) Map of the nonlinear losses excited by the nonlinear propagation of a Bessel pulse in water with soft input regime. (b) Map of the fluence current of the Bessel pulse propagating in the same regime. (c) Map of the fluence current of a Bessel beam propagating in linear regime. Each map is reported as a function of radius (horizontal axis) and propagation distance (vertical axis).

11(a) displays the map of the time-integrated nonlinear losses $\int_{-\infty}^{\infty} q_{\mathrm{MPA}}(z, r, t) d t$. Only the central peak is involved in the dissipation due to the high order of multiphoton absorption. Figure 11(b) displays the map of the energy density flux. For comparison, the energy density flux in the case of linear propagation of the same input field [Eq. (5)] is shown in Fig. 11(c). The flux exhibits two distinct zones: The flux is negative in the part of the Bessel zone corresponding to an increase of the intensity of the central peak. A positive net flux characterizes the larger distances where the central peak intensity decreases owing to apodization and finite energy content of the input beam. It is clear from the comparison between Figs. 11(b) and 11(c), that the energy flux of the steady Bessel filament is similar to that of a linearly propagating Bessel beam. Steady Bessel filaments are thus sustained by a regular refilling and depletion of the central peak where nonlinear losses take place. This means that steady Bessel filamentation is promoted by organizing the energy reservoir so as to support a regular energy flux as that sustaining the unbalanced nonlinear Bessel beams [23].

\section{E. Continuous and bursty plasma channels formed by steady and unsteady Bessel filaments}

Numerical simulations of the plasma channel generated by Bessel filamentation in water were performed by taking again plasma defocusing and plasma absorption into account in Eq. (2). We investigated both cases of steady and unsteady Bessel filaments by launching a $200 \mathrm{fs}, 527 \mathrm{~nm}$ pulsed Gaussian beam of $0.6 \mathrm{~mm}$ radius at $1 / e^{2}$ into a $5.25^{\circ}$ axicon in either soft or abrupt input conditions; the input energy was set to $6.75 \mu \mathrm{J}$.

In both cases a tenuous plasma channel $(\rho \sim 4-5$ $\times 10^{18} \mathrm{~cm}^{-3}$ ) is generated and ionization is confined only in the central peak due to the high order of the excitation process [Figs. 12(a) and 12(b)]. The electron density is in very good agreement with the recent measurements of the refraction index changes within filaments in water [15] by a quantitative refractive shadowgraphy method [58]. Similarly to (a)

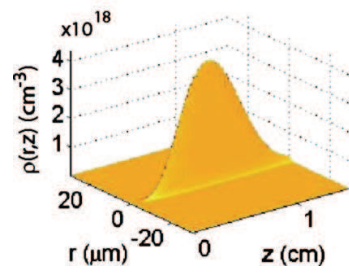

(b)

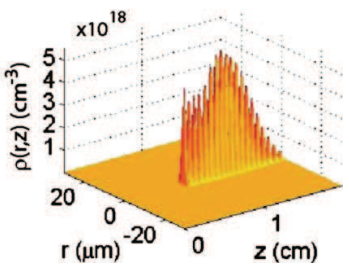

FIG. 12. (Color online) Maps of the plasma density as a function of radius and propagation distance. The plasma channel in (a) was excited by a $200 \mathrm{fs}$ Bessel pulse propagating in water in soft input conditions; plasma channel in (b) was generated by the same Bessel pulse in abrupt input conditions.

the cases of steady Bessel filaments in air, the plasma channel generated by the family of nonlinear unbalanced Bessel beams excited in this regime [Fig. 12(a)] is a continuous and regular trace. On the other hand, the recursive pulse splitting events and beam refocusing occurring in the unsteady Bessel filament generate a plasma channel in the form of a periodic row of electron bursts.

\section{F. Effects of asymmetry}

All simulations performed in this paper assume that the beam preserves its cylindrical symmetry along propagation. In an experiment, the input beam will be unavoidably asymmetric: (i) The beam will be inhomogeneous and (ii) it may exhibit intensity gradients that could result in a self-bending effect [59-61] eventually breaking up the beam into smaller beams fanning away from the axis. However, this effect is not critical in the case of axicon-focused laser pulses. Indeed,
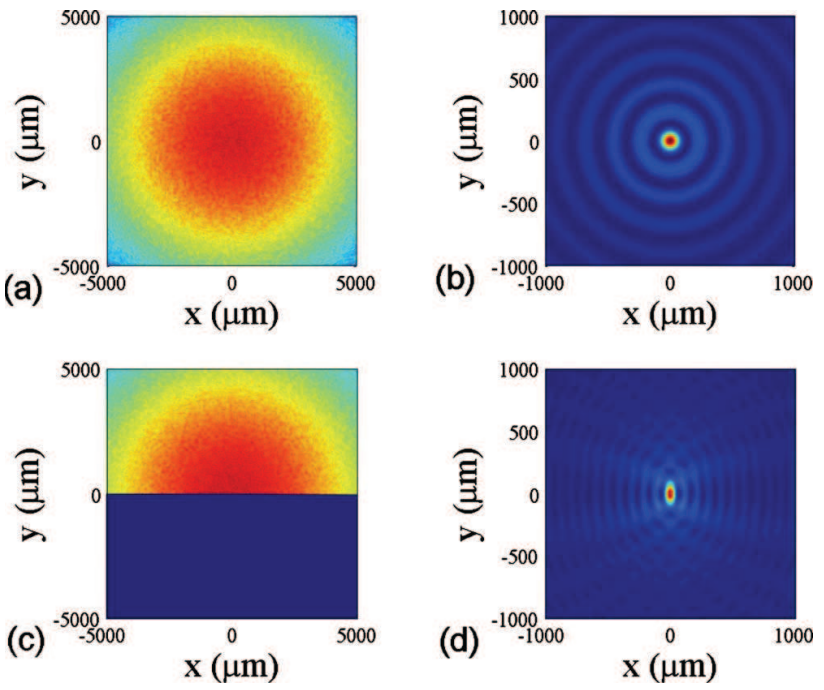

FIG. 13. (Color online) Evolution of intense inhomogeneous Gaussian beam profiles focused in air by an axicon of cone angle $\gamma=0.5^{\circ}$. (a) Input beam profile; $w_{0}=1 \mathrm{~cm}, E_{\text {in }}=2 \mathrm{~mJ}$. The amplitude ratio between inhomogeneity and peak electric field is $10 \%$. (b) Fluence profile at $z=2 \mathrm{~m}$. (c) One-half Gaussian input laser beam with $10 \%$ inhomogeneity. (d) Fluence profile at $z=2 \mathrm{~m}$. 
beam break-up did not occur in any of our previous experiments in condensed media [26,27,51]. In addition, we investigated the possible occurrence of self-bending from numerical simulations where extreme asymmetry can be forced. Figure 13 shows that focusing by an axicon the same Gaussian input beam as that used for the simulations of Bessel filamentation in air with added inhomogeneities [Fig. 13(a)] still leads to a nearly perfect nonlinear Bessel beam [Fig. 13(b)]. The effect of the axicon prevails over that of inhomogeneity and the beam remains centered on axis, even if the axicon cone angle is an order of magnitude smaller than that in Table I. This is still valid in the case of a strong beam asymmetry. Figure 13(c) shows the result of a numerical experiment in the spirit of Ref. [59] where one-half of a Gaussian laser beam (with 10\% inhomogeneity) is used as an input condition and focused by an axicon in a Kerr medium. After $2 \mathrm{~m}$, the propagated beam is centered on axis and the effect of the strong asymmetry is only visible on the interference pattern in the Bessel rings. This shows that Bessel filaments are rather robust structures which do not undergo beam break-up and multiple filamentation so easily as standard filaments. In this respect, Bessel filaments are more suitable to convey large energies over extended distances.

\section{CONCLUSION}

In conclusion, we have identified three different regimes of Bessel filamentation occurring with ultrashort laser pulses when the Gaussian beam is focused by an axicon. The weakly nonlinear Bessel filamentation regime does not involve a significant role of multiphoton absorption and plasma generation, leading to a purely optical interaction with the medium. In contrast, the steady and the unsteady
Bessel filamentation regimes both lead to a strong MPA and plasma generation over long distances.

Unsteady Bessel filaments have common features with filaments generated from pulsed Gaussian beams: In particular, they easily lead to refocusing cycles and a periodic illumination of the sample. However, the energy deposition to matter is far from optimal in standard filaments since the beam still contains a large amount of energy when it is no longer refocused. Unsteady Bessel filaments take advantage of the preorganized energy flux to sustain long-distance illumination of the sample. Steady Bessel filaments ensure a continuous illumination of the medium. The transition between unsteady and steady filamentation is not only governed by the Bessel angle, which determines the periodicity of the energy refilling process, but also by the relevance of multiphoton absorption. Large MPA promotes steady Bessel filamentation since it can damp the increase of intensity that would occur without losses by self-focusing after a refilling stage. Bessel beams therefore produce filaments with high contrast and longer illumination than Gaussian beams with similar power, due to the optimal use of the energy contained in the reservoir. Bessel filaments are foreseen to be better than standard filaments for applications in modern optical technology such as nonlinear microscopy or longitudinal micromachining.

\section{ACKNOWLEDGMENTS}

Fruitful discussions with A. Mysyrowicz's team are gratefully acknowledged. Two of the authors (P.D.T., D.F.) acknowledge financial support from Consorzio Nazionale Interuniveristario per le Scienze fisiche della Materia (CNISM), progetto INNESCO. One of the authors (P.D.T.) acknowledges support from Marie Curie project STELLA, Contract No. MEXC-CT-2005-025710.
[1] G. A. Askar'yan, Sov. Phys. JETP 15, 1088 (1962).

[2] A. Braun, G. Korn, X. Liu, D. Du, J. Squier, and G. Mourou, Opt. Lett. 20, 73 (1995).

[3] A. Couairon and A. Mysyrowicz, Phys. Rep. 441, 47 (2007).

[4] M. Mlejnek, E. M. Wright, and J. V. Moloney, Opt. Lett. 23, 382 (1998).

[5] A. Couairon, S. Tzortzakis, L. Bergé, M. Franco, B. Prade, and A. Mysyrowicz, J. Opt. Soc. Am. B 19, 1117 (2002).

[6] J. H. Marburger, Prog. Quantum Electron. 4, 35 (1975).

[7] A. Couairon, Phys. Rev. A 68, 015801 (2003).

[8] W. Liu, S. L. Chin, O. Kosareva, I. S. Golubtsov, and V. P. Kandidov, Opt. Commun. 225, 193 (2003).

[9] S. Polyakov, F. Yoshino, and G. Stegeman, J. Opt. Soc. Am. B 18, 1891 (2001).

[10] E. Gaižauskas, A. Dubietis, V. Kudriašov, V. Sirutkaitis, A. Couairon, D. Faccio, and P. Di Trapani, Self-focusing: Past and Present Fundamentals and Prospects, Topics in Applied Physics, Vol. 114, edited by R. W. Boyd, S. G. Lukishova, and Y. R. Shen (Springer, New York, 2008), Chap. 20.

[11] V. Kudriašov, E. Gaižauskas, and V. Sirutkaitis, J. Opt. Soc. Am. B 22, 2619 (2005).

[12] S. Tzortzakis, L. Sudrie, M. Franco, B. Prade, A. Mysyrowicz,
A. Couairon, and L. Bergé, Phys. Rev. Lett. 87, 213902 (2001).

[13] A. Couairon, L. Sudrie, M. Franco, B. Prade, and A. Mysyrowicz, Phys. Rev. B 71, 125435 (2005).

[14] A. Dubietis, A. Couairon, E. Kučinskas, G. Tamošauskas, E. Gaižauskas, D. Faccio, and P. Di Trapani, Appl. Phys. B: Lasers Opt. 84, 439 (2006).

[15] S. Minardi, A. Gopal, M. Tatarakis, A. Couairon, G. Tamošauskas, R. Piskarskas, A. Dubietis, and P. D. Trapani, Opt. Lett. 33, 86 (2008)

[16] S. Tzortzakis, M. A. Franco, Y.-B. André, A. Chiron, B. Lamouroux, B. S. Prade, and A. Mysyrowicz, Phys. Rev. E 60, R3505 (1999).

[17] M. Mlejnek, M. Kolesik, J. V. Moloney, and E. M. Wright, Phys. Rev. Lett. 83, 2938 (1999).

[18] M. Kolesik and J. V. Moloney, Opt. Lett. 29, 590 (2004).

[19] A. Dubietis, E. Kučinskas, G. Tamošauskas, E. Gaižauskas, M. A. Porras, and P. Di Trapani, Opt. Lett. 29, 2893 (2004).

[20] A. Dubietis, E. Gaižauskas, G. Tamošauskas, and P. Di Trapani, Phys. Rev. Lett. 92, 253903 (2004).

[21] W. Liu, J.-F. Gravel, F. Théberge, A. Becker, and S. L. Chin, Appl. Phys. B: Lasers Opt. 80, 857 (2005). 
[22] D. Faccio, A. Averchi, A. Lotti, P. Di Trapani, A. Couairon, D. Papazoglou, and S. Tzortzakis, Opt. Express 16, 1565 (2008).

[23] M. A. Porras, A. Parola, D. Faccio, A. Dubietis, and P. Di Trapani, Phys. Rev. Lett. 93, 153902 (2004).

[24] M. Erdelyi, Z. L. Horvath, G. Szabo, Z. Bor, F. Tittel, J. Cavallaro, and M. Smayling, J. Vac. Sci. Technol. B 15, 287 (1997).

[25] J. Amako, D. Sawaki, and E. Fujii, J. Opt. Soc. Am. B 20, 2562 (2003).

[26] P. Polesana, D. Faccio, P. Di Trapani, A. Dubietis, A. Piskarkas, A. Couairon, and M. A. Porras, Opt. Express 13, 6160 (2005).

[27] P. Polesana, A. Dubietis, M. A. Porras, E. Kučinskas, D. Faccio, A. Couairon, and P. Di Trapani, Phys. Rev. E 73, 056612 (2006).

[28] D. E. Roskey, M. Kolesik, J. V. Moloney, and E. M. Wright, Opt. Express 15, 9893 (2007).

[29] E. Gaižauskas, E. Vanagas, V. Jarutis, S. Juodkazis, V. Mizeikis, and H. Misawa, Opt. Lett. 31, 80 (2006).

[30] A. Couairon and L. Bergé, Phys. Plasmas 7, 193 (2000).

[31] S. Tzortzakis, L. Bergé, A. Couairon, M. Franco, B. Prade, and A. Mysyrowicz, Phys. Rev. Lett. 86, 5470 (2001).

[32] A. Couairon, G. Méchain, S. Tzortzakis, M. Franco, B. Lamouroux, B. Prade, and A. Mysyrowicz, Opt. Commun. 225, 177 (2003).

[33] G. Méchain, A. Couairon, M. Franco, B. Prade, and A. Mysyrowicz, Phys. Rev. Lett. 93, 035003 (2004).

[34] G. Méchain, A. Couairon, Y.-B. André, C. D’Amico, M. Franco, B. Prade, S. Tzortzakis, A. Mysyrowicz, and R. Sauerbrey, Appl. Phys. B: Lasers Opt. 79, 379 (2004).

[35] G. Méchain, C. D’Amico, Y.-B. André, S. Tzortzakis, M. Franco, B. Prade, A. Mysyrowicz, A. Couairon, E. Salmon, and R. Sauerbrey, Opt. Commun. 247, 171 (2005).

[36] A. Couairon, M. Franco, G. Méchain, T. Olivier, B. Prade, and A. Mysyrowicz, Opt. Commun. 259, 265 (2006).

[37] B. Prade, M. Franco, A. Mysyrowicz, A. Couairon, H. Buersing, B. Eberle, M. Krenz, D. Seiffer, and O. Vasseur, Opt. Lett. 31, 2601 (2006).

[38] L. Sudrie, A. Couairon, M. Franco, B. Lamouroux, B. Prade, S. Tzortzakis, and A. Mysyrowicz, Phys. Rev. Lett. 89, 186601 (2002).

[39] D. Faccio, A. Matijosius, A. Dubietis, R. Piskarskas, A. Varanavičius, E. Gaižauskas, A. Piskarskas, A. Couairon, and P. Di Trapani, Phys. Rev. E 72, 037601 (2005).

[40] A. Couairon, E. Gaižauskas, D. Faccio, A. Dubietis, and P. Di
Trapani, Phys. Rev. E 73, 016608 (2006).

[41] F. Bragheri, D. Faccio, A. Couairon, A. Matijosius, G. Tamošauskas, A. Varanavičius, V. Degiorgio, A. Piskarskas, and P. Di Trapani, Phys. Rev. A 76, 025801 (2007).

[42] T. Brabec and F. Krausz, Phys. Rev. Lett. 78, 3282 (1997).

[43] R. H. Stolen, J. P. Gordon, W. J. Tomlison, and H. A. Haus, J. Opt. Soc. Am. B 6, 1159 (1989).

[44] E. T. J. Nibbering, G. Grillon, M. A. Franco, B. S. Prade, and A. Mysyrowicz, J. Opt. Soc. Am. B 14, 650 (1997).

[45] E. Yablonovitch and N. Bloembergen, Phys. Rev. Lett. 29, 907 (1972).

[46] D. McGloin and K. Dholakia, Contemp. Phys. 46, 15 (2005).

[47] M. Kolesik, E. M. Wright, and J. V. Moloney, Phys. Rev. Lett. 92, 253901 (2004).

[48] D. Faccio, M. A. Porras, A. Dubietis, F. Bragheri, A. Couairon, and P. Di Trapani, Phys. Rev. Lett. 96, 193901 (2006).

[49] D. Faccio, M. A. Porras, A. Dubietis, G. Tamošauskas, E. Kučinskas, A. Couairon, and P. Di Trapani, Opt. Commun. 265, 672 (2006).

[50] D. Faccio, A. Averchi, A. Couairon, A. Dubietis, R. Piskarskas, A. Matijosius, F. Bragheri, M. A. Porras, A. Piskarskas, and P. Di Trapani, Phys. Rev. E 74, 047603 (2006).

[51] P. Polesana, A. Couairon, D. Faccio, A. Parola, M. A. Porras, A. Dubietis, A. Piskarskas, and P. Di Trapani, Phys. Rev. Lett. 99, 223902 (2007).

[52] C. D'Amico, A. Houard, M. Franco, B. Prade, A. Mysyrowicz, A. Couairon, and V. T. Tikhonchuk, Phys. Rev. Lett. 98, 235002 (2007).

[53] V. B. Gildenburg and N. V. Vvedenskii, Phys. Rev. Lett. 98, 245002 (2007).

[54] S. Akturk, C. D'Amico, M. Franco, A. Couairon, and A. Mysyrowicz, Phys. Rev. A 76, 063819 (2007).

[55] C. D'Amico, A. Houard, S. Akturk, Y. Liu, J. L. Bloas, M. Franco, B. Prade, A. Couairon, V. T. Tikhonchuk, and A. Mysyrowicz, New J. Phys. 10, 013015 (2008).

[56] J. Philip, C. D’Amico, G. Chériaux, A. Couairon, B. Prade, and A. Mysyrowicz, Phys. Rev. Lett. 95, 163901 (2005).

[57] V. Garces-Chavez, D. McGloin, H. Melville, W. Sibbett, and K. Dholakia, Nature (London) 419, 145 (2002).

[58] A. Gopal, S. Minardi, and M. Tatarakis, Opt. Lett. 32, 1238 (2007).

[59] G. A. Swartzlander, Jr., H. Yin, and A. E. Kaplan, Opt. Lett. 13, 1011 (1988).

[60] A. E. Kaplan, JETP Lett. 9, 33 (1969).

[61] M. S. Brodin and A. M. Kamuz, JETP Lett. 9, 351 (1969). 